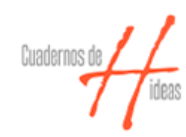

Cuadernos de $\mathrm{H}$ ideas

ISSN: 2313-9048

cuadernosdehideas@perio.unlp.edu.ar

Universidad Nacional de La Plata

Argentina

\title{
Empobrecimiento de las clases medias y la persistencia de la pobreza en México $\left(^{\star}\right)$
}

Millán, Henio

Empobrecimiento de las clases medias y la persistencia de la pobreza en México ${ }^{*}$ )

Cuadernos de H ideas, vol. 12, núm. 12, 2018

Universidad Nacional de La Plata, Argentina

DOI: https://doi.org/10.24215/23139048e016

Esta obra está bajo una Licencia Creative Commons Atribución-NoComercial-SinDerivar 4.0 Internacional. 


\title{
Artículos
}

\section{Empobrecimiento de las clases medias y la persistencia de la pobreza en México $\left(^{*}\right)$}

\author{
Impoverishment of the middle classes and the persistence of \\ poverty in Mexico. \\ Henio Millán hmillan@cmq.edu.mx \\ El Colegio Mexiquense, México
}

Cuadernos de H ideas, vol. 12, núm. 12, 2018

Universidad Nacional de La Plata, Argentina

Recepción: 18 Abril 2018

Aprobación: 10 Julio 2018

DOI: https://

doi.org/10.24215/23139048e 016
Resumen: La incidencia de la pobreza en México muestra niveles similares a los de 1992. Sin embargo, su evolución exhibe dos períodos claramente diferenciados: en el primero (1996-2006) declinó permanentemente, mientras en el segundo (2006-2014) aumentó. A través de un ejercicio de descomposición, se prueba que el efecto redistributivo cambió de naturaleza en los dos períodos: en el primero, las transferencias eran entre no pobres, mientras que en el último ocurren de pobres a no pobres.

Palabras clave: Pobreza, Descomposición, Clases Medias, Desarrollo.

Abstract: Mexican headcount of poverty shows similar levels of 1992. Nevertheless, its evolution exhibits two clearly different periods: in the first one (1996-2006) it declined permanently, as in the second one (2006-2014) it raised. Through a decomposition exercise it is proved that redistribution effect changed of nature between two periods: in the first, transfers were among the non poor, as in the second these occurred from poor to non poor.

Keywords: Poverty, Decomposition, Middle Classes, Development.

Prácticamente desde 1997, la política social mexicana se ha concentrado de forma casi exclusiva en el combate a la pobreza. La estrategia ha consistido en un conjunto de transferencias condicionadas que iniciaron con el Programa de Educación, Salud y Alimentación (PROGRESA)(1) y continuaron con otras denominaciones.(2) Estas transferencias se volvieron el pilar de la lucha contra la transmisión intergeneracional de la pobreza. En esta herencia perpetua radicaba el verdadero problema de este flagelo social. Según esta concepción —avalada por los aportes de Sen-,(3) obedecía fundamentalmente a la falta de capacidades, señaladamente a la formación de capital humano, que impedía a las personas y sus descendientes abandonar esa condición.

Por tal razón, los programas gubernamentales que se han ejecutado en tal dirección, apuntan a la formación de capital humano mediante el pago del costo de oportunidad que, para una familia pobre, significa que sus hijos estudien: no se trata de que el monto sea suficiente para que la población beneficiaria deje de ser pobre, sino de que los infantes y jóvenes permanezcan en la escuela y asistan periódicamente a los centro de salud. La transferencia persigue desmantelar el principal motivo del abandono o el descuido escolar: el trabajo o la mendicidad infantil. Por ello, consiste en una beca que cubre los ingresos que los niños o los jóvenes dejan de percibir al dedicarse de tiempo completo a la educación. En este sentido, 
está condicionada a la asistencia escolar y a las visitas predeterminadas a inspecciones sanitarias.

Los resultados de esa política se consignan en la siguiente gráfica, que refleja la incidencia de la pobreza, a partir de los datos que suministra el Coneval.(4)

Gráfica 1.

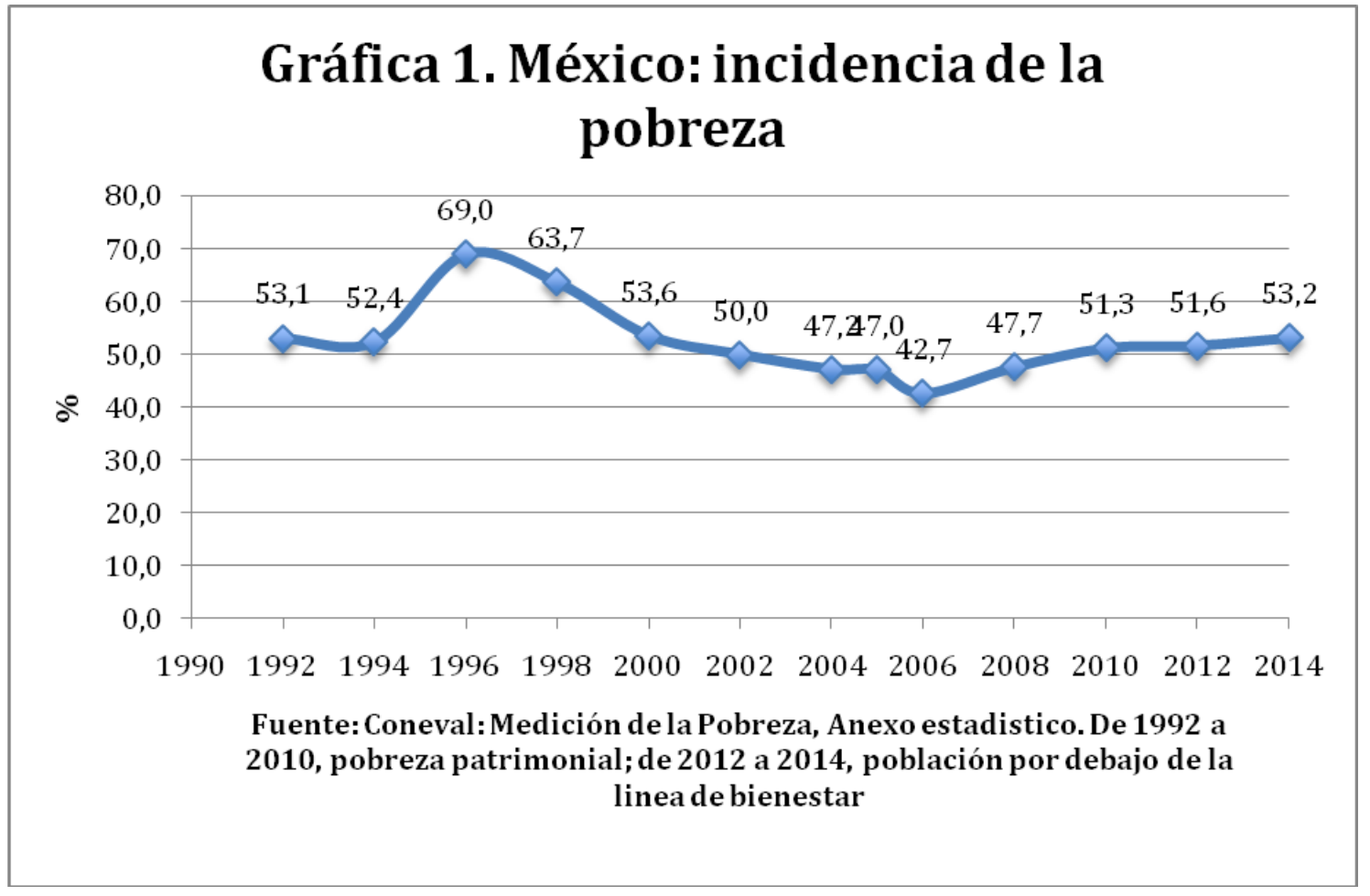

Lo que debería de llamarnos la atención de la gráfica son dos hechos: primero, que a pesar de que el gobierno mexicano ha dedicado cantidades considerables de recursos fiscales a transferencias condicionadas, la incidencia de la pobreza es prácticamente igual a la de 1992. Segundo, que esta evolución puede separarse, con fines analíticos,en dos períodos claramente distintos: 1996-2006 y 2006-2014. En el primero la pobreza declina continuamente, para subir persistentemente en el segundo, hasta alcanzar las cifras que mostraba al comenzar la década de los noventas.De esta forma, la pobreza parece haber alcanzado un piso en 2006 y un techo en 1992 y 2014. ¿Significan estos dos umbrales los límites que demarcan en rango de la pobreza? No lo sé y amerita un estudio detallado y orientado a contestar esa pregunta. Lo que los datos nos dicen es que en estos dos períodos la incidencia de la pobreza registra comportamientos contrarios, a pesar de estar atendida por una misma política social: la estrategia fue exitosa inicialmente, pero después es derrotada por otras realidades, a partir de 2006. Una de estas realidades fue el crecimiento económico, que ha menguado desde entonces, como exhibe la siguiente gráfica: 


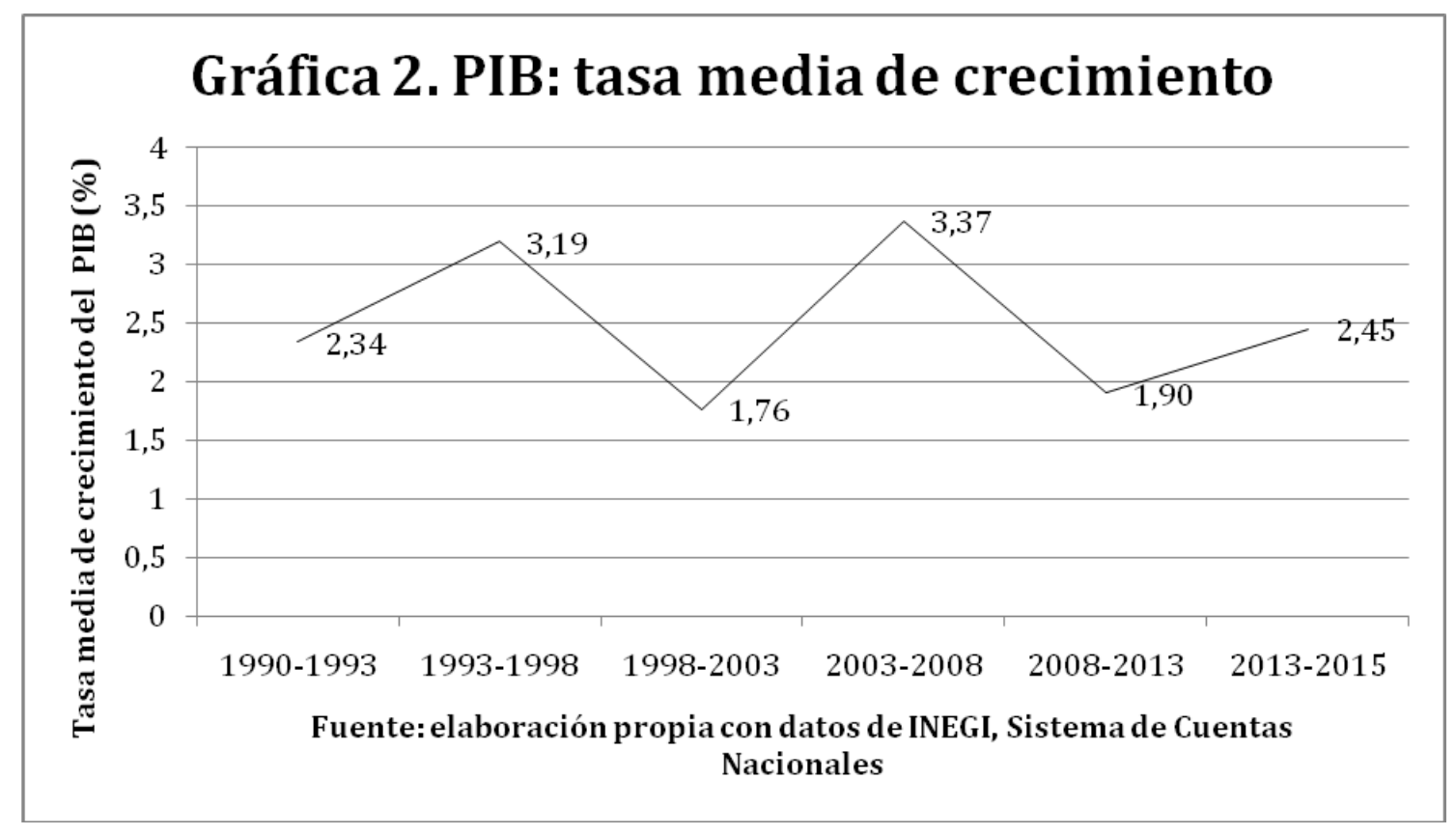

La lógica implícita de aquellos programas es que, estimulados por las becas y los paquetes de salud y alimentación, los niños y jóvenes permanecerían más años en el sistema escolar. Y así ha sido. No obstante, se esperaba que esa mayor permanencia los habilitara para aprovechar las oportunidades de empleo y de inversión que ofrece el mercado. Pero esas oportunidades dependen del crecimiento económico. Cuando éste es insuficiente, tales oportunidades no existen, a pesar de que haya más jóvenes capaces de aprovecharlas. Un examen detallado de este fracaso se puede ver en Millán.(5)

Hay otro aspecto que debe serresaltado de la gráfica 1: mientras la incidencia de la pobreza declinó persistentemente, después de haber repuntado durante la crisis de 1995, no sucedió lo mismo tras la crisis de 2008, a pesar de que ambas fueron de magnitud similar. Esta última fue seguida de una recuperación del PIB que desentona con la conducta en los indicadores de pobreza. Después del colapso de la mitad de los noventa, la incidencia emprendió un patrón declinante; en cambio, la de 2008 fue seguida por un dinamismo económico en el que el fenómeno continuóaumentado. De esta forma, tenemos dos crisis de igual magnitud, pero con recuperaciones que han tenido impactos contrarios sobre la pobreza.

Esto sugiere que se debe encontrar otra forma de inspeccionar las bases más inmediatas del comportamiento de la pobreza. Una candidata para tal propósito es la descomposición de sus variaciones en dos componentes: el efecto crecimiento y el efecto redistribución. El objetivo de estas líneas es examinar los dos períodos a la luz de esta descomposición, con el propósito de detectar cuáles son los factores que explican las conductas diferenciadas que registró la pobreza.Para conseguirlo, se organizan de la siguiente manera: en la primera sección, se examinan las bases conceptuales de la descomposición de la pobreza a través de un método alternativo al de Datt-Ravallion y de Kakwani.(6) Se pretende eludir los problemas que 
ambas técnicas suscitan y, al mismo, tiempo, preparar el camino para un examen más avanzado. En la segunda sección se descomponen los cambios en la incidencia de la pobreza que ha registrado México en los dos períodos mencionados. La tercera trata de identificar cuál es el papel de la redistribución del ingreso entre pobres y clases medias, así como entre éstas y ricos, en las diferencias entre los dos períodos, así como aquel que puede ser atribuido al crecimiento económico. La última, como es costumbre, simplemente concluye.

\section{El efecto crecimiento y el efecto redistribución: la propuesta de Millán}

El efecto ingreso o crecimiento puede ser definido como la variación que registra un indicador de pobreza (incidencia, intensidad y severidad)(7) que puede ser atribuido a la variación en el ingreso. En este sentido, se trata del cambio en aquella cuando se altera el ingreso medio de una sociedad y se mantiene constante la distribución del ingreso. El efecto redistribución, por su parte, es la variación en la pobreza atribuible a alteraciones en el reparto del ingreso. Por tanto, se mide por la mutación que experimenta la pobreza cuandocambia la distribución y el ingreso medio se mantiene constante. De esta forma, la variación total del indicador de pobreza debería de ser igual a la suma de los dos efectos(8):

$$
\Delta I=E C+E R
$$

Donde $\Delta I$ es la variación en el indicador de pobreza (en este caso, la incidencia); $E C$, el efecto crecimiento; y $E R$, el efecto redistribución.

Los métodos tradicionales para descomponer las variaciones de la pobreza son dos: el de Datt-Ravallion y de Kakwani.(9) Ambos estiman el efecto crecimiento mediante variaciones en el ingreso medio y una curva de Lorenz constante; y el efecto redistribución, exactamente al revés: el ingreso medio dado, mientras la curva de Lorenz cambia entre dos años determinados. Sin embargo, el Datt-Ravallion arroja un conjunto de resultados que para muchos, entre ellos Kakwani, resultan cuestionables. En primer lugar, incorpora un residuo $(\mathrm{R})$ y la ecuación anterior se transforma en la siguiente:

$$
\Delta I=E C+E R+R(1)
$$

En segundo término, este residuo puede ser tan grande que a menudo sobrepasa a uno u ambos efectos. Además, no es simétrico: el resultado de la descomposición no es el mismo cuando la estimación se efectúa desde el año inicial que cuando se realiza a partir del período final.(10)

Estos "defectos" condujeron a Kakwani a emprender otro método para corregirlos. En esencia, consiste en anular el residuo a través de promedios interanuales de cada uno de los efectos:

$$
E R_{t, t-1}=\frac{E R_{t}+E R_{t+1}}{2}
$$




$$
E C_{t, t-1}=\frac{E C_{t}+E C_{t+1}}{2}
$$

La solución ha despertado críticas a causa de su carácter arbitrario: no deriva de una definición de los efectos, a pesar de que responde a un conjunto axiomático que ambos deben de cumplir, porque para muchos -incluidos Datt y Ravallion- el residuo no es descomponible. Y, además, es la forma usual de desvanecer las diferencias entre las derivadas de una curva y las de sus tangentes; es decir, entre las pendientes de ambos lugares geométricos.

Ante la polémica entre los autores de ambos métodos, Millán(11) ideó un método alternativo que, sin recurrir al artificio de los promedios interanuales, genera resultados exactos y sin residuo y, al mismo tiempo, simétricos. Las ecuaciones que definen el efecto crecimiento y el efecto redistribución son las siguientes:

$$
\begin{gathered}
E C=\gamma_{1}\left(\beta_{2}-\beta_{1}\right) \\
E R=\beta_{2}\left(\gamma_{2}-\gamma_{1}\right)
\end{gathered}
$$

Donde $\gamma$ es la relación entre el ingreso medio de la sociedad $(\boldsymbol{X})$ y el ingreso medio de los pobres (W) del período i-ésimo:

$$
y=\frac{X}{W}
$$

De esta forma, $r=\frac{x}{w}$ da cuenta de la relación entre dos ingresos medios: el de la sociedad en su conjunto y el de los pobres. Admite, por lo tanto, desigualdades entre ambos conglomerados y se finca de forma exclusiva en los promedios, sin reparar en las disparidades de los mismos. Lo que interesa es la desigualdad entre los pobres y no pobres. Por eso, sus variaciones $\left(\gamma_{2}-\gamma_{1}\right)$ son un indicador de los cambios en la pobreza atribuibles a la distribución del ingreso entre ambos núcleos sociales: un alza de $\gamma$ indica que la sociedad se hace más rica y que los pobres se rezagan ante ese aumento de riqueza, ya sea porque su ingreso medio crece menos que proporcionalmente o porque, francamente, declina. La baja opera en sentido contrario.

Por otro lado, $\beta$ es la proporción del ingreso total de los pobres $\left(Y_{p}\right)$ en el ingreso total o nacional $(Y)$, del período i-ésimo

$$
\beta=\frac{Y_{p}}{Y}
$$

Las variaciones de $\beta$, dado $\gamma$, dan cuenta del efecto crecimiento o ingreso. Un aumento significará que el ingreso de los pobres se eleva como proporción del ingreso nacional, porque existen un contingente más amplio de personas pobres; y no porque éstos enriquecen. Lo contrario sucede con una baja. Es, por tanto, un indicador de cómo afecta el crecimiento del ingreso en las variaciones de la pobreza. 
Con esta guía es posible descomponer las variaciones en la pobreza a partir de la distribución del ingreso que reporta la Encuesta Nacional de Ingresos y Gastos del INEGI y de los datos que sobre la pobreza aporta el Coneval. Primero se realizará esta operación para los períodos de crisis y auges; y después, se optará por una visión de más largo plazo: la que corresponde a los lapsos que nos interesan.

\section{Descomposición de las variaciones de la pobreza en México: crisis y recuperaciones}

Desde 1992, México ha encardo tres crisis de importancia: la de 1995, la de 2002 y la de 2009. La primera y la última han sido similares, si juzgamos por el abatimiento del PIB, mientras que la de inicio del milenio representó sólo una caída moderada. La intención de este apartado es presentar la forma en que el crecimiento económico y la distribución del ingreso se han comportado en las crisis y las recesiones, a fin de determinar el significado preciso de los efectos desde una perspectiva más amplia. Los resultados se ofrecen en la siguiente gráfica:

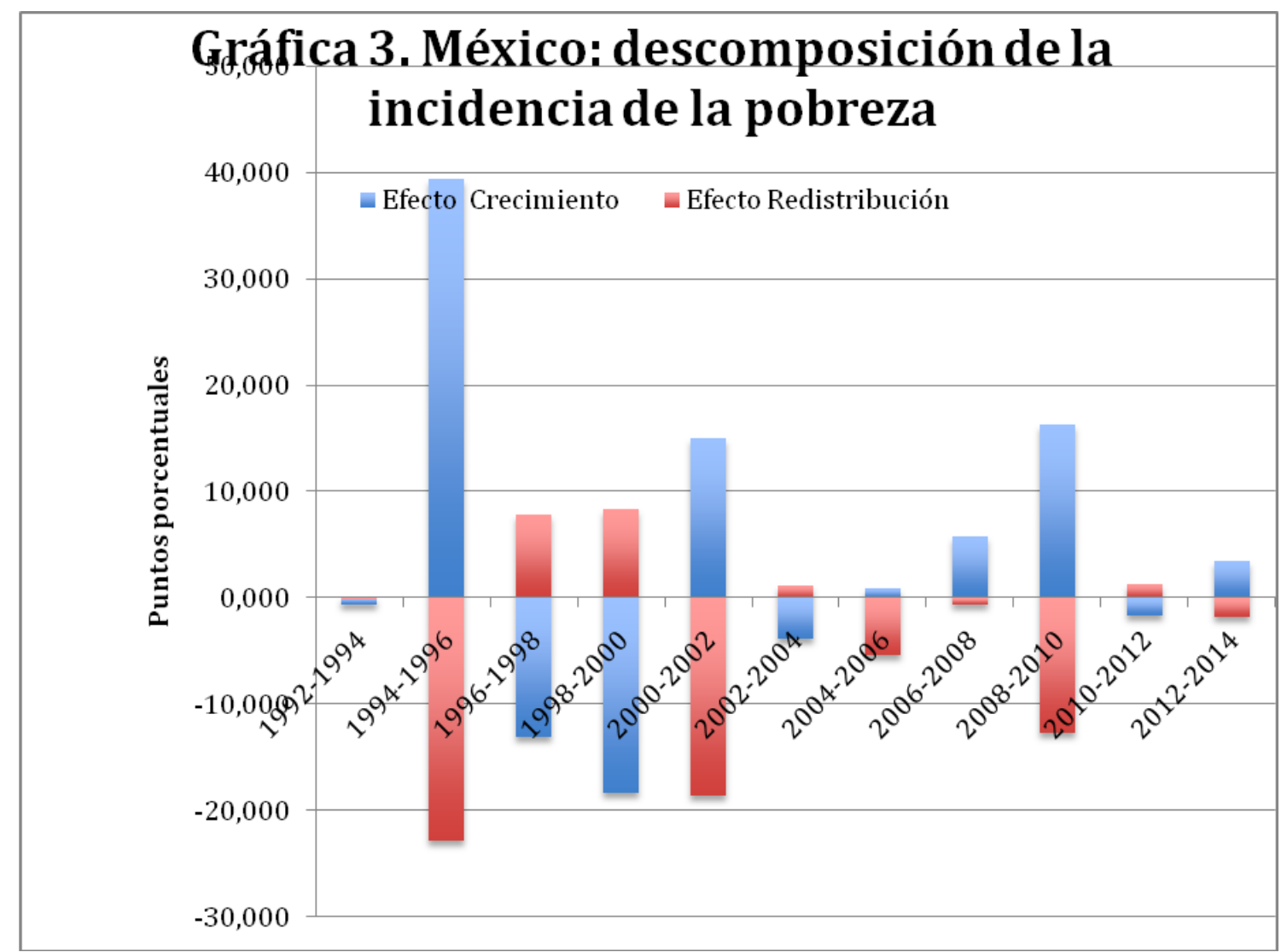

La gráfica es elocuente en dos aspectos fundamentales: en primer término, se aprecia que los efectos son invariablemente de signo contrario.(12) Ello significa que cuando uno de los dos disminuye la pobreza, el otro la aumenta. Más específicamente, cuando la economía mexicana crece, esta expansión reduce la incidencia de la pobreza; pero el modelo de desarrollo suscita fuerzas que tienden a concentrar el ingreso, 
de tal forma que se activa el efecto redistribución en sentido contrario, provocando una aumento de la pobreza. En cambio, cuando la economía se abate, se implanta un mecanismo que impulsa la pobreza al alza, pero tal tendencia se ve amortiguada por una mejoría en la distribución del ingreso. En este sentido, el efecto redistribución opera como un freno en el abatimiento de la pobreza en las expansiones económicas, así como un amortiguador en las recesiones.

El patrón inverso entre crecimiento y distribución del ingreso se puede apreciar en la siguiente gráfica:

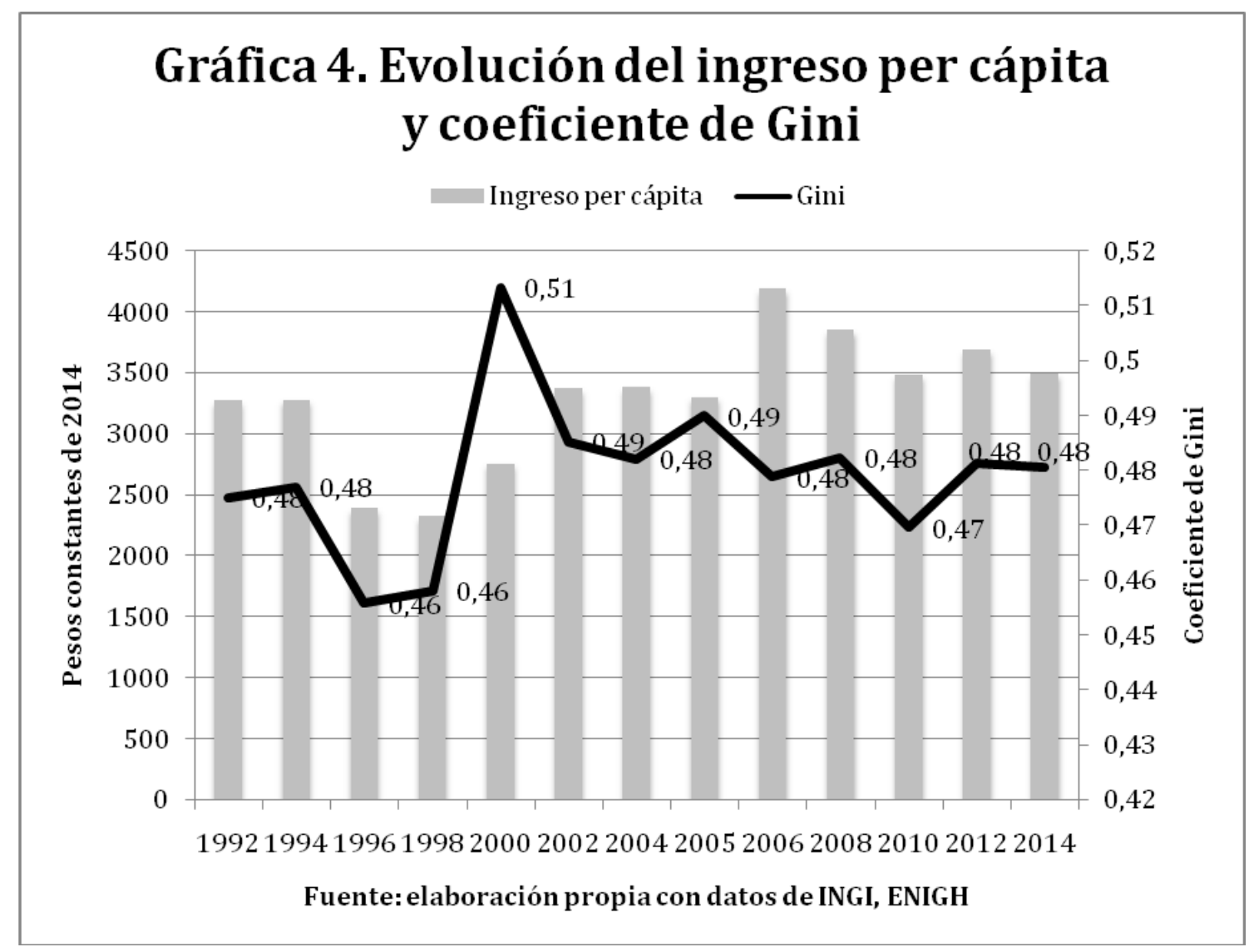

Como se puede observar, en términos generales, el modelo de desarrollo acusa un rasgo en que el crecimiento económico y la redistribución progresiva del ingreso se comportan en sentido inverso. Esta tendencia es más clara cuando se observan períodos más amplios que los anuales. De esta forma, un mayor ingreso per cápita familiar se ve acompañado por una concentración del ingreso; y lo contrario sucede cuando aquel declina. Ello nos advierte que los frutos del crecimiento tienden a concentrarse entre los que más tienen y a excluir a los más pobres. Pero también, éstos son los que sufren menos las recesiones porque los pobres, además de esta condición, son marginados, en el sentido más cabal del término: no participan de los frutos del crecimiento, pero también son los relativamente menos afectados cuando la economía declina, porque sus actividades suelen operar al margen de las grandes dinámicas del mercado, especialmente cuando éstas se determinan por los vaivenes de los sectores exportadores. 
El segundo aspecto que exhibe la gráfica 3 es que, con la excepción del período 2000-2002, el efecto crecimiento predomina sobre el impacto redistributivo. Es decir, es la capacidad de expansión de la economía la que exhibe un mayor potencial para abatir los índices de pobreza, aunque - evidentemente - la distribución también está llamada a jugar un papel en esta dirección. La importancia de este hecho - sobre todo al combinarse con el primero - radica en que si, como se ha visto, el crecimiento se ha enlentecido, las posibilidades para abatir la pobreza se ven seriamente disminuidas; pero adicionalmente, su potencial mengua en la medida en que el crecimiento genera fuerzas concentradoras del ingreso que menguan la cantidad de pobres que, sin ese efecto, habrían abandonado la pobreza si tales fuerzas no estuvieran presentes cuando la economía se expande.

De esta forma, encontramos en el debilitamiento del crecimiento una primera explicación de por qué la pobreza persiste; pero a ella se debe agregar que su potencial se ve reducido por el efecto redistribución, que aumenta la pobreza cuando el efecto crecimiento la reduce, aunque sea en escala cada vez menor. Por tanto, las reducciones de la pobreza estimuladas por el crecimiento se ven frenadas por una concentración del ingreso en estas fases, mientras los aumentos acicateados por declinaciones en el ingreso ingresoper cápita la amortiguan con una relativa mejoría en la distribución. Existe, pues, un juego de contrapesos entre estas dos variables que hacen que la pobreza persista como fenómeno de largo plazo, aunque acepta variaciones durante temporadas relativamente largas como las que mediaron entre 1996 y 2006 y, más recientemente, entre 2008 y 2014, aunque en sentido contrario.

\section{La descomposición en largo plazo y el papel de las clases medias}

Una forma de acercarnos un poco más a las causas que, en el largo plazo, determinan la persistencia de la pobreza, consiste en examinar más de cerca los determinantes de los dos efectos que explican sus variaciones. Según la ecuación (2), el efecto crecimiento está determinado por el nivel inicial $d_{\gamma_{1}}$ y la variación de $\beta$. Hay una forma de relacionar las dos variables. De acuerdo a la ecuación (5),

$$
\beta=\frac{Y_{p}}{Y}=\frac{W P}{X P O B}=\frac{1}{Y} I
$$

Donde I es la incidencia de la pobreza; P, el número de pobres; y POB, la población total.

De esta forma, vemos que la distribución del ingreso entre pobres y el conjunto de la sociedad, medida por la participación de los primeros en el ingreso nacional, depende de la incidencia y de la proporción entre los ingresos medios de la sociedad y los de los pobres. Es decir, contiene un componente estrictamente distributivo $\left(\frac{1}{\bar{r}}=\frac{w}{x}\right)$ y otro poblacional $\left[I=\left(\frac{P}{\rho 08}\right)\right]$ 
. La definición de efecto crecimiento nos pide mantener constante la distribución y hacer variar el ingreso medio:

$$
\begin{aligned}
& \dot{X} \neq 0 \\
& \dot{X}=\dot{W}
\end{aligned}
$$

En este sentido, opera como contrafactual (una constante); y por ello, se ofrece como un dato, un evento del pasado, bajo la forma de su valor inicial.

$$
\Delta \beta=\frac{1}{\gamma_{1}} \Delta I
$$

Es decir, las variaciones de $\beta$ reflejan cambios en la incidencia de la pobreza, sin ninguna alteración entre la relación de ingresos medios (de la sociedad y de los pobres). De otra forma: un aumento de aquella variable mostrará una mayor participación de los pobres en el ingreso nacional, no porque su ingreso haya mejorado con relación al del conjunto social, sino simplemente porque hay más pobres; o mejor: porque el número de pobres crece a un ritmo superior al de la población:

$$
\dot{P}>P \dot{O} B
$$

¿Cuál es el significado de esta proposición? Que un número indeterminado de las personas que inicialmente no eran pobres, ingresaron al contingente de los pobres. Es decir, se presenta una depauperación de las clases medias.(13) En sentido contrario, si $\beta$ desciende, el número de pobres crece menos que la población, lo cual significa que una parte de los que originalmente vivían en pobreza, abandonaron esa condición.

Con el efecto redistribución ocurre lo contrario: la definición nos demanda alterar la distribución del ingreso y mantener constante el ingreso medio:

$$
\begin{aligned}
& \dot{X}=0 \\
& \dot{X} \neq \dot{W}
\end{aligned}
$$

En este sentido, $\mathrm{X}$ opera como una constante y las variaciones de $\gamma$ sólo puede provenir de cambios en W.

$$
\begin{gathered}
\gamma=\frac{X}{W}=X W^{-1} \\
\Delta \gamma=-\frac{X}{W^{2}} \Delta W=-\gamma \dot{W} \\
\dot{\gamma}=-\dot{W}
\end{gathered}
$$


Específicamente, la tasa de cambio de las remuneraciones medias de los pobres es igual, pero con signo contrario, a la tasa de variación distributiva entre los ingreso medios de la sociedad y aquellas remuneraciones. Por tanto, cuando los ingresos medios de los pobres aumentan, mejora la distribución del ingreso a favor de ellos; y lo contrario sucede cuando disminuyen. En este sentido una variación de $\gamma$ puede ser interpretada como un cambio en sentido contrario en los ingresos medios de los pobres. Un aumento se traducirá en una disminución de esos emolumentos; y una caída, en un ascenso de los mismos. Para que tales movimientos puedan afectar la incidencia de la pobreza, es necesario que actúen sobre el número de pobres, si consideramos que:

$$
\begin{gathered}
W=\frac{Y_{p}}{P} \\
P=\frac{Y_{p}}{W}
\end{gathered}
$$

En la medida en quex $\dot{x}=0$, un cambio regresivo en distribución sólo puede provenir de una declinación de W. En la descomposición, tal mutación debería provocar un aumento en número de pobres, porque supone que $\dot{W}>\dot{Y}_{p}^{\dot{p}}$. Sin embargo, esta proposición necesita transparentarse para que adquiera un sentido lógico.

En el caso del efecto crecimiento es claro cuál es el mecanismo que conduce a engrosar la filas de los pobres: la declinación generalizada, aunque no necesariamente proporcional, del ingreso medio. Pero no sucede lo mismo con el efecto redistribución.

Expliquemos el punto: supongamos que la incidencia cambia cuando el ingreso medio es constante; y además, se altera la distribución; tal y como lo exige la definición de este efecto. Un aumento de la incidencia no puede ser atribuido a una transferencia desde los contingentes de los pobres hacia el de los no pobres. Efectivamente, la redistribución se vuelve más regresiva y los pobres acentúan su pobreza. Ello agrava la intensidad de quienes la padecen, pero deja inalterado el nivel de incidencia, porque ningún persona sale de - ni entra a - las filas de los pobres. Entonces, se tiene una $\mathrm{W}$ más baja y una distribución más regresiva del ingreso, pero no se ha efectuado ningún trastrocamiento en la incidencia. Los pobres son ahora más pobres (aumento en la intensidad), pero la cantidad de ellos sigue siendo la misma. ¿Significa esto que la lógica del efecto redistribución deja de funcionar? No.

En realidad, la declinación de $\mathrm{W}$ es una causa más que un efecto de cambios distributivos entre los no pobres. Supongamos que $Y_{p}=100 ; P=5 y W=20$. También que se produce transferencia de ingresos desde un no pobre a otro más rico.(14) Antes de este acto, el primero registraba un ingreso de 40; si la trasferencia es de 30, entonces se reduce a 10 e incurre en la pobreza. Se ha producido una cambio regresivo en la distribución, pero ahora el número de pobres ha aumentado en una persona. Tras el movimiento, los datos han cambiado: $Y_{p}=110 ; P=6 \mathrm{yW}=18.33$ . Es decir, ha bajado el ingreso medio de los pobres a consecuencia de 
que el nuevo integrante incurre con una remuneración menor (10) a la mediaper cápita anterior $(\mathrm{W}=20)$. Esto tiende a abatir el promedio (ingreso per cápita $=W$ ) de los pobres. Por tanto, el menoscabo de la distribución del ingreso opera entre los no pobres; pero también, debido a que ingresan en la filas de los pobres con un ingreso inferior al promedio que previamente registraban estos últimos, W declina y, además, nos advierte que tal menoscabo impacta fuertemente a las clases medias, en la medida en que su ingreso es inferior al de los pobres, cuando se vuelven parte de ellos.

Por supuesto que tales variaciones dejan inalterado el ingreso medio de la sociedad: la reducción en W se ve compensada por el aumento del promedio de ingresos entre los no pobres, debido a que sin cambiar el monto de estos últimos, hay un no pobre menos:

$$
Y=Y_{p}+Y_{n p}
$$

Donde np son los no pobres; y $Y_{n p}$ es su ingreso total.

$$
\begin{array}{r}
X=\frac{Y_{p}}{P O B}+\frac{Y_{n p}}{P O B} \\
\frac{Y_{p}}{P O B}=\frac{W P}{P O B}=W I \\
\frac{Y_{n p}}{P O B}=\frac{W^{\prime} \cdot N P}{P O B}=W^{*}(1-I)
\end{array}
$$

Donde $\mathrm{W}^{\prime}$ es ingreso medio de los no pobres. Sustituyendo:

$$
\begin{gathered}
X=W I+W^{\prime}(1-I) \\
\Delta I=-\Delta(1-I) \\
-\Delta W=\Delta W^{\prime} \quad(12)
\end{gathered}
$$

Para finalizar, dos cosas. En primer lugar, si cuando hay un aumento de la incidencia debido a una regresión en la distribución y ésta obedece a transferencias entre no pobres, no sucede lo mismo cuando la incidencia disminuye debido a una distribución más equitativa. En este último caso, el traspaso de recursos de no pobres a pobres puede perfectamente abatir la incidencia de la pobreza, como se espera de la lógica tradicional del efecto redistribución. En nuestros términos, significa un impulso alcista de W suscitado por la transferencia de recursos desde la trinchera de los no pobres. Ello se traducirá en un descenso dey.

Y por último, aunque la discusión anterior puede resultar engorrosa y en efecto, así lo es -, sus conclusiones enriquecen el argumento sobre la persistencia de la pobreza. Por un lado, sabemos que las variaciones en la incidencia por efecto crecimiento responden a los vaivenes en el mismo sentido en el ingreso medio de la sociedad. Las clases medias se ven afectadas por tales cambios y engrosan las filas de los pobres cuando declina, mientras éstos reciben más ingresos y salen de la pobreza, cuando 
aumenta. Aquí no hay secreto alguno; sólo el que vincula la mengua del crecimiento con las dificultades para abatir los índices de la pobreza.

Pero, por el otro, el efecto redistributivo encierra misterios más difíciles de desentrañar: la redistribución regresiva del ingreso afecta la incidencia a través de cambios en el reparto entre los no pobres, y tales mutaciones operan mediante una declinación del ingreso medio de los pobres, porque el ingreso de los nuevos contingentes a la fila de quienes la conforman se realiza mediante ingresos inferiores al promedio previo que registraban antes de las variaciones en la distribución. La progresiva opera en el sentido tradicional: los pobres reciben más ingresos de los no pobres y pueden emigrar de la condición de pobreza.

¿Cómo afectan estas conclusiones a la idea de la persistencia de la pobreza? En primer término, la mengua del crecimiento hace más difícil abatirla, como se ha argumentado. Pero, además, cuando los no pobres devienen pobres por una redistribución regresiva entre ellos, el hecho de que tal transformación se realice con ingresos inferiores al promedio previo de los pobres, implica que cuando la distribución mejora (cuando la economía empeora) es necesario suministrarles un ingreso por la vía tradicional (no pobres transfieren a los pobres) mucho mayor que el antiguo promedio de los pobres. Ello implica que el cambio distributivo en un sentido progresivo debe ser radical, de gran impacto y de gran monto. Esto, como se aprecia en la gráfica 3, no sucede en México. La única excepción es el período 2000-2002, cuando el efecto redistribución superó el efecto crecimiento.

De esta forma, nos encontramos con: a) un crecimiento menguado, que además se frena por el efecto redistribución; y b) mejoras distributivas cuando las hay - que son muy débiles para impactar sensiblemente la pobreza. De esta forma, se conjugan dos potentes fuerzas que contribuyen a que la pobreza persista, a pesar de los esfuerzos gubernamentales por combatirla a través de transferencias condicionadas. El cuadro siguiente resume cómo estas tendencias se han manifestado en México, en dos períodos claramente diferenciados: el primero es de abatimiento de la pobreza; el segundo, de aumento continuado; es decir, los lapsos bajo nuestro estudio: 


\section{Cuadro 1.}

Variación porcentual de los determinantes de los efectos crecimiento y redistribución

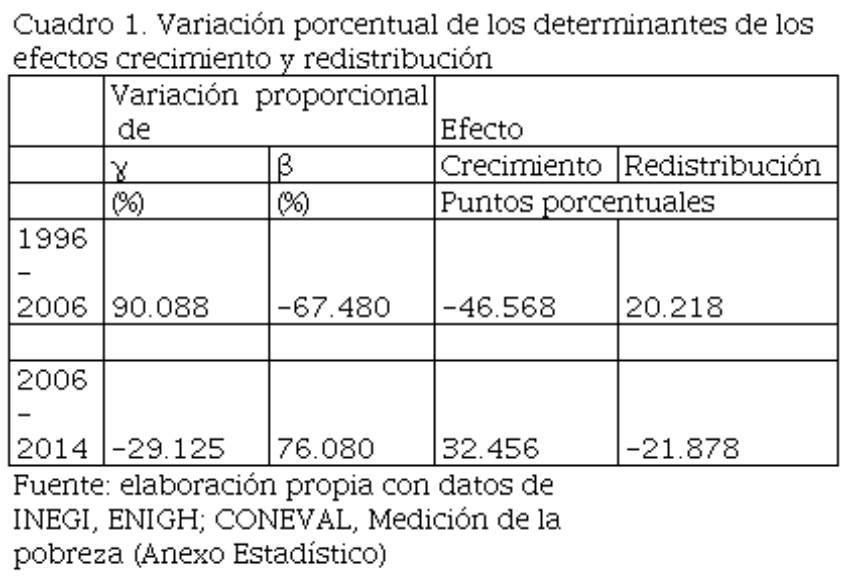

Fuente: elaboración propia con datos de INEGI, ENIGH; CONEVAL, Medición de la pobreza (Anexo Estadístico)

Los datos nos advierten que, en primer término, el crecimiento económico perdió su capacidad para abatir la pobreza en el segundo período, en virtud - precisamente - de la mengua que ha sufrido durante este lapso. Ahora ha contribuido a su aumento (como lo atestigua el signo positivo que registra durante 2006-2014). En este sentido, la responsabilidad se ha desplazado hacia la redistribución del ingreso, que ha registrado en esta segunda etapa una tendencia de largo plazo a ser más equitativa, especialmente si el referente es 2005, el año previo al arranque de esta fase (gráfica 3). Pero, en segundo término, el período en cuestión reproduce el patrón de inferioridad numérica de la redistribución, cuando se trata de incidir en la pobreza.

Pero también nos dicen que en el lapso más reciente se ha producido un cambio estructural en el efecto redistribución: mientras entre 1996 y 2006, la concentración del ingreso operaba mediante la redistribución entre los no pobres (aumento significativo de $\gamma$ ), en el segundo período, ha actuado desde los no pobres hacia los pobres. Enla medida en que el primer lapso coincide - en términos generales - con la puesta en marcha del Tratado de Libre Comercio de América del Norte (TLCAN), que reimpulsó aceleradamente la exportación de bienes de consumo duradero, especialmente los de la industria automotriz, es posible explicar la primera redistribución como un desplazamiento de clases medias menos calificadas por otras más habilitadas para incrustarse en una dinámica económica intensiva en conocimiento. De esta manera, se vieron empobrecidas por la inserción en el mercado internacional. En el segundo,tales movimientos cesan o se hacen sentir con menor intensidad, porque esos sectores líderes encuentran una mano de obra relativamente más calificada y más abundante, que abarcaba a segmentos importantes de los pobres. (15)

A ello, sin duda, ha contribuido la política de trasferencias, porque si bien es cierto que ha fracasado para interrumpir la transmisión 
intergeneracional, también lo es que sí ha sido una factor de abatimiento del rezago escolar. Ello ha generado un conocimiento base más difundido a través de una expansión de la educación secundaria, que ha facilitado su incorporación a los sectores exportadores, en condiciones salariales menos onerosas para las empresas, pero - al mismo tiempo — más lucrativa para algunos segmentos pobres (Millán, 2013).(16) De esta forma, se produjo una mejor distribución del ingreso hacia estos núcleos sociales.

\section{Conclusiones}

En resumen, la persistencia de la pobreza en México encuentra su raíz de ser en la forma en que funciona el modelo de desarrollo; pero, en tiempo recientes, se han gestado rasgos que la hacen más evidente, en virtud de que el crecimiento económico ha perdido su fuelle para abatir la pobreza, simplemente porque se ha desacelerado dramáticamente, mientras el otro efecto que podría hacerlo - el redistributivo - y que ahora lo releva en esa misión, continúa siendo muy débil, precisamente porque descansa en transferencias de no pobres a pobres, que están sujetas a restricciones institucionales involucradas con las estructuras del poder.

\section{Notas}

$\left.{ }^{*}\right)$ El artículo que se presenta es una versión modificada y actualizada del capítulo de un libro que actualmente se encuentra en prensa sobre trampas de la pobreza. Ahonda en aspectos de la descomposición de los cambios en la pobreza que conducen a entender mejor cómo sus contingentes se han nutrido de clases medias

(1)Sedesol. Programa de Educación, Salud y Alimentación. Progresa, México, Sedesol, 1998. Recuperado de: https://www.gob.mx/cms/uploads/attachment/ file/79893/1998--.pdf

(2)Los programas que le siguieron fueron: "Oportunidades" que, además de incluir a los alumnos de secundaria, se amplió para incorporar a los de bachillerato y los contingentes urbanos en pobreza extrema; y, recientemente, "Prospera. Programa de inclusión social", que agrega una salida productiva a quienes han sido beneficiados con las transferencias condicionadas.

(3) Sen, Amartya. "Capacidad y bienestar" en AmartyaSen y Martha Nussbaum (comp.) La calidad de vida, México, FCE, 1998.

(4) El Coneval es el Consejo Nacional para la Evaluación de la Política Social. Tiene dos funciones: la primera es evaluar los programas sociales del gobierno mexicano; la segunda, medir la pobreza.Coneval. Anexo estadístico. Medición de la pobreza 2010-2014, 2015. Recuperado de: http://www.coneval.org.mx/Medicion/ EDP/Paginas/Evolucion-de-las-dimensiones-de-la-pobreza-1990-2014-.aspx

(5) Millán, Henio. La pieza faltante. El combate a la pobreza y el crecimiento económico en México. México, Porrúa y el Colegio Mexiquense, 2005.

(6) Datt, G., y M. Ravallion. "Growth and redistribution components in poverty measures: A decomposition with applications to Brazil and India in the 1980's”, Journal of Development Economics (38), 1992, 275-295; Kakwani, N.On measuring growth and inequality components of changes in poverty with application to Thailand.School of Economics, The University of South Wales, mimeo, 1997. 
(7) Un examen de las trespuedeencontrarseenLevy y Foster. Por lo pronto, es conveniente aclarar que la incidencia de la pobreza mide cuántos y quiénes son los pobres, mediante el conteo de personas que se encuentran por debajo de una línea de pobreza como proporción de la población total. La intensidad de la pobreza nos dice qué tan pobres son los pobres. La medición cuenta la suma de las brechas entre el ingreso de un pobre individual y su ingreso, como proporción de la línea de la pobreza. La severidad, además de incorporar estas dimensiones, nos dice que tan desiguales son los pobres y otorga a las brechas más grandes una mayor ponderación. Foster, J., J. Greer, and E. Thorbecke: "A Class of DecomposablePovertyMeasures," Econometrica, 52, 1984, 761-766; Levy, S. "La pobreza en México" en Félix Váldez, La pobreza en México. Causas y políticas para superarla, México, ITAM-FCE, 1994.

(8)Bourguignon, F..The Poverty-Growth-Inequality Triangle.Ponencia presentada en el Indian Council forResearch on International EconomicRelations. Nueva Delhi, 4 de febrero, 2004. Disponible en: http://siteresources.worldbank.org/INTPGI/ Resources/342674-1206111890151/15185_ICRIER_paper-final.pdf

(9)Datt-Ravallion (1992) y de Kakwani (1997), Op. Cit.

(10) Mahmoudi, 2001.

(11) Millán, Henio. "Los efectos crecimiento y redistribución: una propuesta metodológica. Ciclo económico y pobreza en México”. El Trimestre Económico, LXXXI, 323, 2014, pp. 657-687.

(12)Recordamos al lector que un signo positivo denota un aumento de la incidencia de la pobreza, y uno negativo, una diminución de este indicador.

(13)Hay que hacer notar que existe la posibilidad de que la tasa de crecimiento de la población pobre sea mayor a la general, simplemente por el hecho de que los pobres tengan más hijos; pero tal posibilidad la descartamos para efecto del análisis.

(14)Esta transferencia puede obedecer a varios motivos. Uno de ellos es, por ejemplo, la inflación. En el fondo, ésta consiste en la trasferencia desde segmentos de ingreso fijo a otros de ingreso variable. El no pobre puede recibir un ingreso fijo (un sueldo), mientras un empresario o comerciante registrará remuneraciones variables.

(15) López-Calva, J. L, y Lustig, Nora. The recent decline of inequality in Latin America: Argentina, Brazil, Mexico and Peru, ECINEQWP, 2009 - 140; World Bank. The Labor Market Story Behind the Latin America's Transformation, Washington, D.C, 2012.

(16) Millán, Henio. En la cuerda floja. Vulnerabildad hacia la pobreza y fragilidad laboral en México, Toluca, El Colegio Mexiquense, 2013.

\title{
Enlace alternativo
}

\author{
http://perio.unlp.edu.ar/ojs/index.php/cps/index (html)
}

